\title{
Helical bunching and symmetry lowering inducing multiferroicity in Fe langasites
}

\author{
L. Chaix, ${ }^{1,2,3,4}$ R. Ballou, ${ }^{2,3}$ A. Cano, ${ }^{5}$ S. Petit, ${ }^{6}$ S. de Brion,,${ }^{2,3}$ J. Ollivier, ${ }^{1}$ L.-P. Regnault, ${ }^{3,7}$ E. Ressouche,,${ }^{3,7}$ E. Constable, ${ }^{2,3}$ \\ C. V. Colin, ${ }^{2,3}$ A. Zorko, ${ }^{8}$ V. Scagnoli, ${ }^{9,10}$ J. Balay, ${ }^{2,3}$ P. Lejay, ${ }^{2,3}$ and V. Simonet ${ }^{2,3}$ \\ ${ }^{1}$ Institut Laue-Langevin, 6 rue Jules Horowitz, 38042 Grenoble, France \\ ${ }^{2}$ Institut Néel, Centre National de la Recherche Scientifique, 38042 Grenoble, France \\ ${ }^{3}$ Université Grenoble Alpes, 38042 Grenoble, France \\ ${ }^{4}$ Stanford Institute for Materials and Energy Sciences, SLAC National Accelerator Laboratory, Menlo Park, California 94025, USA \\ ${ }^{5}$ Centre National de la Recherche Scientifique, Université Bordeaux, ICMCB, UPR 9048, F-33600 Pessac, France \\ ${ }^{6}$ Commissariat à l'Énergie Atomique et aux Énergies Alternatives, Centre de Saclay/DSM/IRAMIS/Laboratoire Léon Brillouin, \\ 91191 Gif-sur-Yvette, France \\ ${ }^{7}$ MEM-MDN, INAC, 38054 Grenoble, France \\ ${ }^{8}$ Jožef Stefan Institute, Jamova c. 39, SI-1000 Ljubljana, Slovenia \\ ${ }^{9}$ Laboratory for Mesoscopic Systems, Department of Materials, ETH Zurich, 8093 Zurich, Switzerland \\ ${ }^{10}$ Paul Scherrer Institute, 5232 Villigen PSI, Switzerland
}

(Received 10 June 2015; revised manuscript received 25 May 2016; published 16 June 2016)

\begin{abstract}
The chiral Fe-based langasites represent model systems of triangle-based frustrated magnets with a strong potential for multiferroicity. We report neutron-scattering measurements for the multichiral $\mathrm{Ba}_{3} M \mathrm{Fe}_{3} \mathrm{Si}_{2} \mathrm{O}_{14}$ $(M=\mathrm{Nb}, \mathrm{Ta})$ langasites revealing new important features of the magnetic order of these systems: the bunching of the helical modulation along the $c$ axis and the in-plane distortion of the $120^{\circ} \mathrm{Fe}$-spin arrangement. We discuss these subtle features in terms of the microscopic spin Hamiltonian and provide the link to the magnetically induced electric polarization observed in these systems. Thus, our findings put the multiferroicity of this attractive family of materials on solid ground.
\end{abstract}

DOI: 10.1103/PhysRevB.93.214419

The quest for novel magnetic orders that promote multiferroicity and strong magnetoelectric coupling drives a considerable amount of current research (see, e.g., [1-4] for recent reviews). In this respect, Fe-based langasites are propitious materials displaying an original "multi-chiral" magnetic order in which a $120^{\circ} \mathrm{Fe}$-spin arrangement undergoes an additional helical modulation [5-8]. In fact, the magnetoelectric effect in these systems has been demonstrated at both static [9] and dynamical levels [10]. In addition, Zhou et al. [11] have reported the emergence of ferroelectricity at the onset of magnetic order. This ferroelectricity has subsequently been confirmed by Lee et al. [12], who have also demonstrated the magnetic-field manipulation of the electric polarization. However, these works report two different ferroelectric axes at zero field and, above all, leave the precise mechanism promoting such a ferroelectricity unidentified.

Magnetically induced ferroelectricity in many multiferroic cycloidal magnets is due to the spin-orbit coupling (SOC) via the so-called inverse Dzyaloshinskii-Moryia (DM) mechanism [13-15]. However, this mechanism is ineffective for the reported helical magnetic structure of the langasites [16,17]. An electric polarization observed in magnetic helices rather than cycloids has been theorized [18], as well as observed experimentally in $\mathrm{RbFe}\left(\mathrm{MoO}_{4}\right)_{2}$ for instance [16,17,19]. However, the invoked mechanisms still rely on the SOC and appear inapplicable in the langasite case. Khomskii, Bulaevskii et al. $[3,20,21]$ have discussed another fundamental mechanism that can generically operate in triangle-based frustrated magnets. The proposed mechanism is independent of SOC as it simply relies, in the language of Mott insulators, on high-order hopping-term contributions that enable the redistribution of the electronic charge. This purely electronic mechanism can be supplemented by a contribution from lattice degrees of freedom [3]. In any case, it requires a distortion of the perfect $120^{\circ}$-spin structure and, despite its generality, has not been verified experimentally so far.

In this paper, we carefully analyze neutron-scattering experiments to revise the magnetic structure of the $\mathrm{Ba}_{3} M \mathrm{Fe}_{3} \mathrm{Si}_{2} \mathrm{O}_{14}$ ( $M=\mathrm{Nb}$, Ta) langasites. We discover additional features indicating the bunching of the helicoidal modulation and significant deviations from the $120^{\circ}$-spin structure in these systems. In order to explain these features, we propose an extended spin Hamiltonian incorporating different magnetic anisotropies. These extra terms prove to be crucial for understanding the magnetically induced ferroelectricity in the $\mathrm{Fe}$ langasites. Furthermore, we find that this ferroelectricity emerges from a magnetoelectric coupling that is equivalent to the Bulaevskii et al. proposal at the phenomenological level.

The Fe langasites crystallize in the nonpolar non-centrosymmetric space group P321, in which the magnetic $\mathrm{Fe}^{3+}$ ions $(S=5 / 2)$ form a triangular lattice of triangles in the $a b$ plane (see Fig. 1). The magnetic order of these systems, observed below the Néel temperature $\mathrm{T}_{N}$, has been rationalized within the spin Hamiltonian [6,7,22-24]:

$$
\mathcal{H}=\sum_{(i j)} J_{i j} \mathbf{S}_{i} \cdot \mathbf{S}_{j}+\sum_{(i j)_{\Delta}} D\left(\mathbf{S}_{i} \times \mathbf{S}_{j}\right)_{z} .
$$

Here the first term takes into account the exchange interactions, where the sum runs over the spin pairs connected by different paths shown in Fig. 1(a). The nearest- and nextnearest-neighbor interactions $J_{1}$ and $J_{2}$ in the $a b$ plane are both antiferromagnetic. This generates magnetic frustration within the $\mathrm{Fe}$ triangles and causes the $120^{\circ}$ spin structure. Among the three antiferromagnetic exchange couplings between adjacent $a b$ planes, the strongest one is $J_{3}$ or $J_{5}$ depending on 
(a)
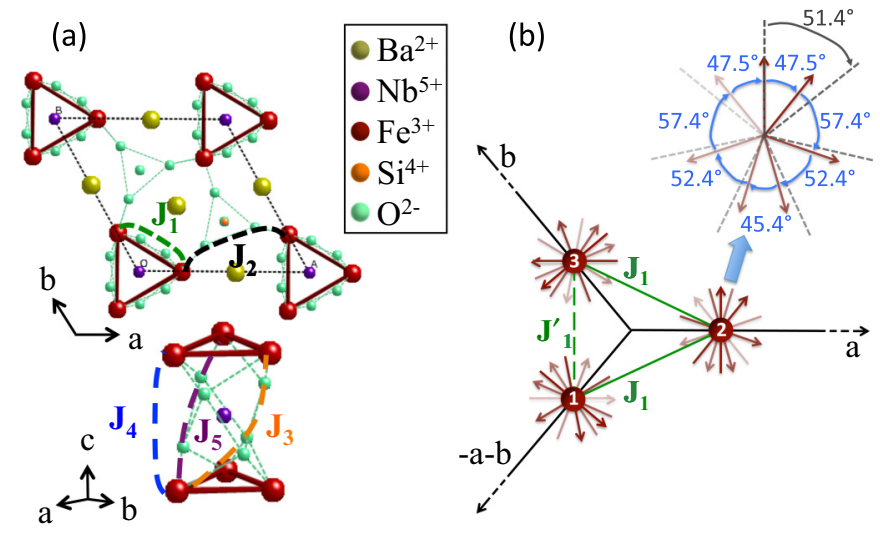

FIG. 1. (a) $\mathrm{Ba}_{3} \mathrm{NbFe}_{3} \mathrm{Si}_{2} \mathrm{O}_{14}$ crystallographic structure in the $a b$ plane and along the $c$ axis, with five magnetic exchange paths $J_{1}$ to $J_{5}$. (b) Schematic representation of the revisited magnetic structure projected along the $c$ axis. The threefold axis is broken leading to different $J_{1}$ interactions (case of an isosceles triangle represented). The bunching of the helices in the $\mathrm{Nb}$ compound is reported through the successive angles between consecutive magnetic moments along $c$, deviating from the regular angle $2 \pi \tau=51.4^{\circ}$.

the structural chirality. These couplings generate the helical modulation of the $120^{\circ}$ structure along the $c$ axis. The propagation vector of this modulation depends on the balance between the interplane interactions $J_{3}, J_{4}$, and $J_{5}$. The second term in Eq. (1), where the sum runs over intratriangle spins, corresponds to the additional DM interactions with the DM vector $(D)$ along the $c$ axis, which is allowed by symmetry. This DM term restricts the spins to the $a b$ plane and favors one intratriangle chirality as observed experimentally $[22,23,25]$. Alternatively, a weak easy-axis single-ion anisotropy can have similar effect [26].

We have revised this structure by carrying out single-crystal neutron-scattering measurements on two members of the $\mathrm{Fe}$ langasite family with $\mathrm{Nb}$ or Ta on the $1 a$ Wyckoff site. The two single crystals, grown by floating-zone method in an image furnace [9], display opposite structural chiralities [25]. The experiments were done on two spectrometers installed at the Institut Laue Langevin: the IN5 time-of-flight spectrometer using unpolarized neutrons, and the CRG-CEA IN22 tripleaxis spectrometer, equipped with CRYOPAD, using polarized neutrons and allowing longitudinal polarization analysis. The dynamical structure factors were measured on IN5 at $1.5 \mathrm{~K}$, for both crystals in rotation about the $a$ zone axis, with an incident wavelength of $4 \AA$. The $\mathrm{Nb}$ compound was also measured on IN22 at $2 \mathrm{~K}$ with a final wave vector fixed to $2.66 \AA^{-1}$ and at zero-energy transfer in order to get a quantitative determination of the elastic structure factors of the magnetic satellites. The crystal was oriented with the $a$ zone axis vertical so as to probe the $\left(b^{*}, c^{*}\right)$ scattering plane. Two types of measurements were carried out using different monochromator-analyzer configurations: Heusler-Heusler (H-H) and Graphite-Heusler (G-H). The former allows us to discriminate the magnetic and chiral contributions from the nuclear one, whereas the latter increases the incident neutron flux and allows us to determine the chiral scattering from an unpolarized beam [7], hence with a larger flux.

The intensity maps at zero-energy cut measured on IN5 for the $\mathrm{Nb}$ and Ta compounds are shown in Figs. 2(a) and 2(b), respectively. The Néel temperatures of these compounds are nearly identical ( $27 \mathrm{~K}$ for $\mathrm{Nb}$ and $28 \mathrm{~K}$ for $\mathrm{Ta}$ ), while the propagation vectors are $\vec{\tau}=(0,0,0.1429)$ and $(0,0,0.1385)$, respectively $[9,27]$. The latter is obtained from the position of the magnetic satellites at $Q=|\vec{H} \pm \vec{\tau}|$ in Fig. 2. In addition to these first-order satellites, we clearly observe higher-order satellites at $Q=|\vec{H} \pm 2 \vec{\tau}|$ and $|\vec{H} \pm 3 \vec{\tau}|$ in both compounds suggesting that the helical modulation is distorted.

Polarized neutron measurements with longitudinal polarization analysis on the $\mathrm{Nb}$ compound allowed us to extract the nuclear $\left(\sigma_{N}\right)$, the magnetic $\left(\sigma_{M}\right)$, and the chiral $\left(\sigma_{\mathrm{ch}}\right)$
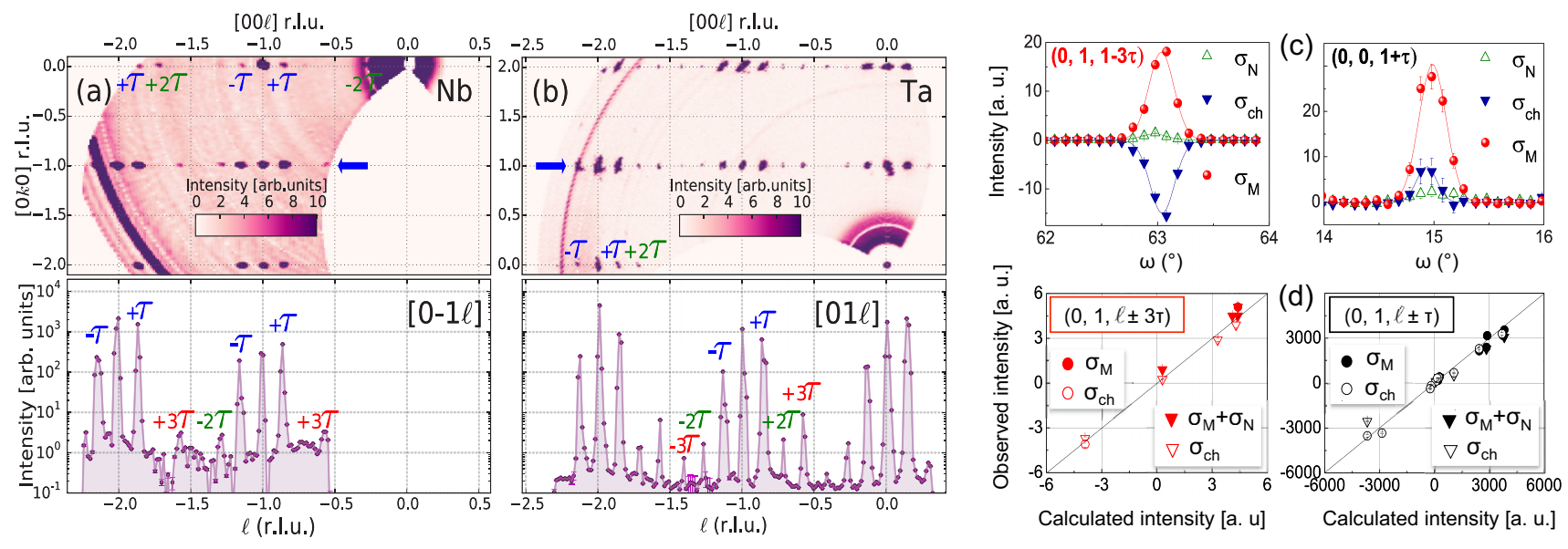

FIG. 2. Zero-energy cut (integrated from -0.1 to $0.1 \mathrm{meV}$ ) from IN5 measurements at $1.5 \mathrm{~K}$, on the $\mathrm{Nb}$ (a) and Ta (b) compounds. The blue arrows on the energy maps indicated the $[0 \pm 1 \ell]^{*}$ directions of the $1 \mathrm{D}-Q$ cuts shown below in vertical log scale. (c),(d) summarize the measurements performed on the $\mathrm{Nb}$ compound at $2 \mathrm{~K}$ on IN22 with polarized neutrons. (c) shows the magnetic (red), nuclear (green), and chiral (blue) elastic contributions from scans obtained by rotating the crystal ( $\omega$ scans) with the H-H configuration. The solid lines are Gaussian fits. The magnetic and chiral contributions measured in the $\mathrm{H}-\mathrm{H}$ (circles) and $\mathrm{G}-\mathrm{H}$ (triangles) configurations for the main $(0,1, \ell \pm \tau)$ and extra $(0,1, \ell \pm 3 \tau)$ satellites, vs the calculated contributions using the model given in the caption of Fig. 3, are displayed in (d). Note that the G-H configuration cannot separate the magnetic from nuclear scattering, but we checked that $\sigma_{M}+\sigma_{N} \approx \sigma_{M}$ for these satellites. 


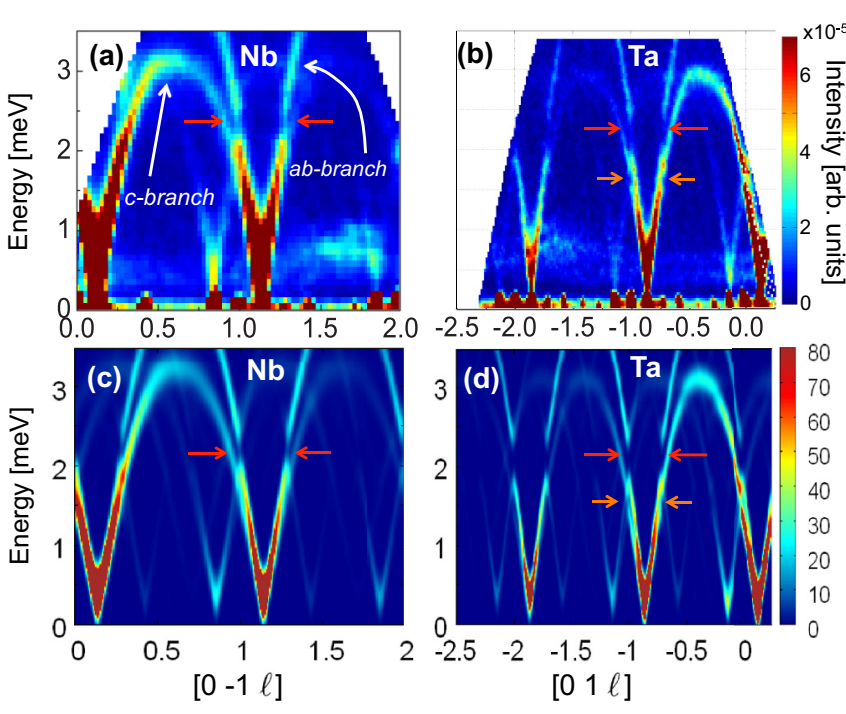

FIG. 3. Spin-waves spectra of $\mathrm{Nb}$ (a) and $\mathrm{Ta}(\mathrm{b})$ measured along the $\left[\begin{array}{llll}0 & -1 & \ell\end{array}\right]^{*}$ and $\left[\begin{array}{lll}0 & 1 & \ell\end{array}\right]^{*}$ directions, respectively, at $1.5 \mathrm{~K}$ on IN5. Below: Spin-waves calculations with the following parameters for the $\mathrm{Nb}(\mathrm{c}) / \mathrm{Ta}(\mathrm{d}): J_{1}=0.85 / 0.85 \pm 0.1 \mathrm{meV}, J_{2}=0.24 / 0.24 \pm$ $0.05 \mathrm{meV}, \quad J_{3}=0.053 / 0.22 \pm 0.03 \mathrm{meV}, \quad J_{4}=0.017 / 0.014 \pm$ $0.05 \mathrm{meV}, J_{5}=0.24 / 0.053 \pm 0.05 \mathrm{meV}, D=-0.028 /-0.028 \pm$ $0.005 \mathrm{meV}$, easy-plane anisotropy $K=0.045 / 0.053 \pm 0.005 \mathrm{meV}$. The red and orange arrows show the spectral weight extinctions.

scattering [25], hence to identify the nuclear and/or magnetic nature of these extra peaks. This analysis reveals that the second-order satellites are mainly of nuclear origin. This is usually attributed to magnetoelastic effects and will not be further discussed. In contrast, the magnetic contribution is dominant in the third-order satellites [see Fig. 2(c)], which confirms the deformation of the magnetic helices.

This deformation allows further explanation of the subtle details in the spin-wave spectrum. As we can see in Fig. 3, the spectra of the $\mathrm{Nb}$ and Ta compounds display two branches emerging from the magnetic satellites [22]. The high-energy branch corresponds to spin components in the $a b$ plane while the low-energy branch is associated to spin components along the $c$ axis. The main features of this spectrum were initially captured by the model of Eq. (1) using the standard HolsteinPrimakov formalism in the linear approximation (see Fig. 4 of Ref. [22]). However, this simple model fails to reproduce a spectral weight extinction observed in the $a b$ branch around $2.3 \mathrm{meV}$ in both compounds, and a tiny extinction around $1.7 \mathrm{meV}$ in the $c$ branch, visible only in the Ta compound (red and orange arrows, respectively, in Fig. 3) [25].

In order to reproduce the deformation of the helices leading to all these features, we have considered a straightforward extension of Eq. (1). We included extra single-ion anisotropy terms with respect to a local twofold $z_{\text {loc }}$ axis, i.e., along $a, b$, and $-a-b$ for the three ions of a triangle, respectively:

$$
H_{\mathrm{sia}}=K \sum_{i} S_{z \mathrm{loc}, i}^{2} .
$$

We first determine the magnetic ground state of the extended Hamiltonian by means of a real-space mean-field energy
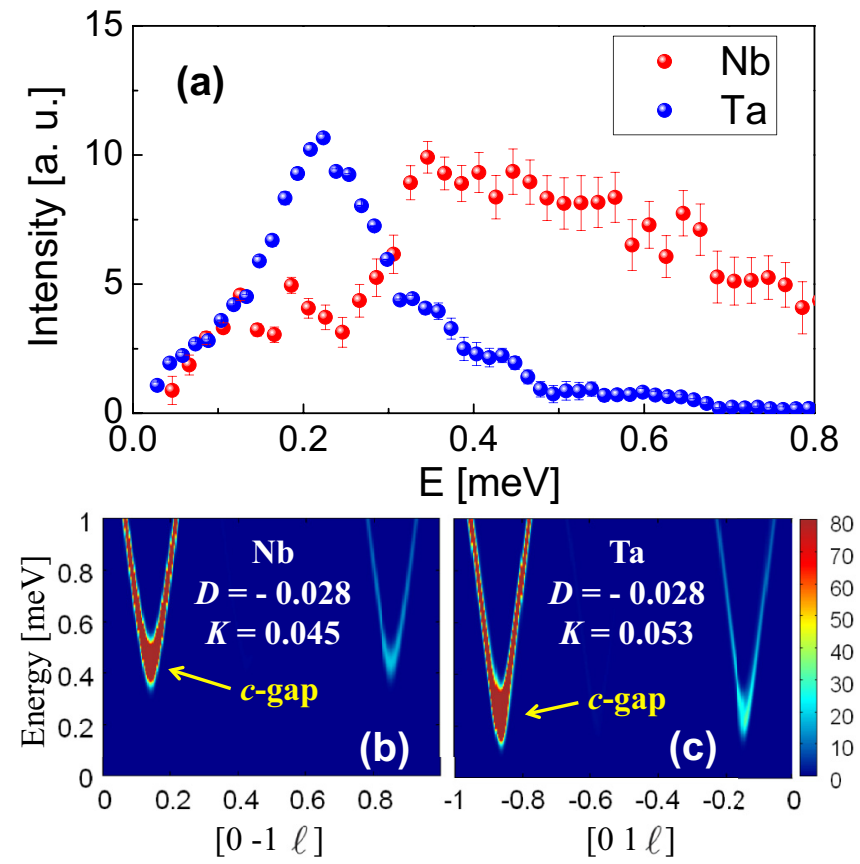

FIG. 4. (a) Neutron-scattering intensity vs the energy at the magnetic satellite position for Ta and $\mathrm{Nb}$, obtained from $1 \mathrm{D}-Q$ cut (integration ranges $\Delta h=\Delta k=\Delta l=0.05$ ) measured on IN5 with a wavelength of $8 \AA$. Below: Calculated scattering intensity of the $c$ branch only showing the $c$ gap of the $\mathrm{Nb}$ (b) and $\mathrm{Ta}$ (c) compounds using the revised model with the parameters of the caption of Fig. 3.

minimization. Then we compute the corresponding magnetic and chiral elastic scatterings for the first- and third-order magnetic satellites, and finally we compute the corresponding spin-wave spectrum [25].

A quantitative determination of the parameters of the revised Hamiltonian can be achieved for the $\mathrm{Nb}$ compound. The intensity of the the first-order $(0, k, \ell \pm \tau)$ and extra $(0$, $k, \ell \pm 3 \tau)$ satellites [Fig. 2(d)] increases with the single-ion anisotropy and agrees with the experiments (see Fig. 2) for $|K|=0.045 \pm 0.05 \mathrm{meV}$. This term is also at the origin of the anticrossings observed in the spin waves (at $2.3 \mathrm{meV}$ for the $a b$ branches and at $1.7 \mathrm{meV}$ for the $c$ branches): these spectral weight extinctions occur at the crossing point of branches with the same symmetry, emerging from the $+\tau$ and $-\tau$ elastic magnetic satellites, which are mixed by the single-ion anisotropy $K S_{z}^{2}[25,28]$. The anticrossings are more pronounced in the Ta case [see Fig. 3(b)] which qualitatively agrees with a higher value of $K$ [25]. Another signature of the presence of tiny anisotropies is a gap in the $c$ branch, which reaches its energy minimum at $\approx 0.4 \mathrm{meV}$ for $\mathrm{Nb}$ [26] and $\approx 0.2 \mathrm{meV}$ for Ta as shown in Fig. 4(a). The variation of this gap is actually opposite for the easy-plane $(K>0)$ and for the easy-axis $(K<0)$ anisotropies. In the latter case, the $c$ gap increases with increasing $|K|$ and is already around $1 \mathrm{meV}$ for $K=0.045 \mathrm{meV}$, which is larger than the values observed experimentally. These values are actually compatible with an easy-plane anisotropy combined with a DM term (with $D<0$ ): the $c$ gap increases when $|D|$ increases and decreases when $K$ increases. The $\mathrm{Nb} c$ gap is reproduced for $D=-0.028 \mathrm{meV}$ and $K=0.045 \mathrm{meV}$ [see Fig. 4(b)]. 
The smaller gap of the Ta compound is in turn compatible with a larger $K$ than in the $\mathrm{Nb}$ one [see Fig. 4(c)]. This analysis yields the extended model given in the caption of Fig. 3 with a set of parameters reproducing the spin waves for the $\mathrm{Nb}$ and $\mathrm{Ta}$ compounds [see Figs. 3(c) and 3(d)]. Moreover, in the $\mathrm{Nb}$ case these parameters are fully compatible with the magnetic structure factors and with the electronspin-resonance measurements [23] that have been reanalyzed carefully [25]. In real space, this revised model globally corresponds to a deviation of the angle between adjacent spins along the helix axis away from the ideal $2 \pi \tau=51.4^{\circ}$ value: It gets smaller near the local easy planes and larger far from them [see Fig. 1(b)]. Thus, we conclude that the magnetic structure of the Fe langasites corresponds to a bunched helix, as reported in some rare-earth intermetallics [29].

Next, we analyze in more detail the first-order satellites along the $\left[\begin{array}{lll}0 & 0 & \ell\end{array}\right]^{*}$ line. These $(0,0, \ell \pm \tau)$ satellites are clearly visible in our data (see Fig. 2), where they display the same temperature dependence as the $(0,1, \ell \pm \tau)$ magnetic ones [25]. In fact, the longitudinal polarization analysis confirms their magnetic origin [see Fig. 2(c)]. The observation of these satellites is very surprising since, in the case of a perfect $120^{\circ}$ spin arrangement within the $\mathrm{Fe}$ triangles, there should be an extinction of the magnetic structure factor along the $\left[\begin{array}{ll}0 & 0\end{array}\right.$ $\ell]^{*}$ line. We note that the forbidden $(0,0, \tau)$ satellite has also been observed by magnetic resonant $\mathrm{x}$-ray diffraction (together with $2 \tau$ and $3 \tau$ satellites in the first Brillouin zone) [30]. This was attributed to a modulated out-of-plane (butterfly) component of the magnetization produced by the in-plane intratriangle DM vector. However, neutrons are blind to this component along $\left[\begin{array}{lll}0 & 0 & \ell\end{array}\right]^{*}$ since they are only sensitive to the magnetic components perpendicular to the scattering vector $Q$. Thus, the observation of these $(0,0, \ell \pm \tau)$ satellites in our neutron experiments clearly demonstrates that the relative orientation of the magnetic moments within the triangles deviates from $120^{\circ}$, hence suggesting that the intratriangle $J_{1}$ interactions are not equivalent. Our neutron data cannot single out whether the distortion of this triangular arrangement is local (helically modulated from planes to planes) or global. However, only the latter case, implying a loss of the threefold symmetry axis of the P321 structure, is compatible with a macroscopic polarization measured along the twofold axis. The loss of the threefold axis was already invoked from Mössbauer experiments and x-ray magnetic diffraction [27,30], and was an important ingredient for explaining the magnetoelectric excitations revealed by means of $\mathrm{THz}$ spectroscopy [10]. In addition, it is compatible with the phonon spectrum [31]. This finding has strong implications on the multiferroic properties of these systems, as we discuss in the following.

The P321 space group of the Fe langasites allows the magnetoelectric coupling of the form

$$
\begin{aligned}
F_{M E}= & \alpha\left\{P_{x}\left(\mathbf{S}_{1}-\mathbf{S}_{2}\right) \cdot \mathbf{S}_{3}\right. \\
& \left.-\frac{1}{\sqrt{3}} P_{y}\left[\left(\mathbf{S}_{1}+\mathbf{S}_{2}\right) \cdot \mathbf{S}_{3}-2 \mathbf{S}_{1} \cdot \mathbf{S}_{2}\right]\right\},
\end{aligned}
$$

where $y$ and $x$ stand for the $a$ axis and its perpendicular direction in the $a b$ plane, and $\mathbf{S}_{1}$ to $\mathbf{S}_{3}$ are the three spins on a triangle (see Fig. 1). This coupling can have a purely electronic origin as discussed in [20] or emerge from magnetostriction effects in the symmetric Heisenberg exchange [3]. In any case, at the phenomenological level, its form is identical to the one discussed in $[3,20,21]$. The presence of such a coupling means that a spin-induced electric polarization has to be expected whenever the spin arrangement deviates from the perfect $120^{\circ}$ structure [32]. This polarization will lie in the $a b$ plane. Thus, the new magnetic structure deduced from our experiments is compatible with the polarization measurements of Lee et al. [12] rather than those of Zhou et al. [11]. This is also in tune with the DFT-based calculations reported in [33], in which a magnetically induced polarization was also obtained in the $a b$ plane due to the reduction of symmetry of the magnetic structure to $\mathrm{C} 2$ '. In this work, however, this reduction was the result of spin-orbit coupling (likely via symmetric anisotropic exchange), and the precise magnetoelectric coupling behind such an electric polarization was not identified.

The enhancement of the electric polarization in the $a b$ plane by the application of a magnetic field has also been reported in [12]. This can be understood on the basis of the above coupling, although its connection with the revised magnetic structure is more subtle. In this case, the $120^{\circ}$ spin structure of each triangle is additionally deformed by the Zeeman coupling, which induces a (weak) ferromagnetic component and thus an extra local contribution to the electric polarization. In the perfect helical structure, however, these individual field-induced electric polarizations coming from the different layers of the helix rotate along the $c$ axis in such a way that they cancel each other. In the bunched helical structure, in contrast, this rotation is modified so that the cancellation is avoided, which eventually enables an overall finite polarization induced by the magnetic field. This will happen even if the initial (zero-field) structure corresponds to a perfect $120^{\circ}$ arrangement within the Fe triangles. Thus, the magnetic-field induced electric polarization essentially relies on the bunching of the helix along the $c$ axis.

By carrying out neutron-scattering experiments, we have identified subtle features in the magnetic order of Fe langasites, with drastic consequences on their properties. The first feature is a bunching of the helical modulation related to single-ion magnetocrystalline anisotropy, and the second one is the distortion of the $120^{\circ}$ spin structure. At the phenomenological level, we have established that the magnetically induced electric polarization at zero and finite magnetic fields results from a generic magnetoelectric coupling that is equivalent to that put forward by Bulaevskii et al. Our study thus sets the basis for understanding the multiferroic properties of Fe langasites representing model-case magnetically induced ferroelectrics in triangle-based frustrated magnets. Finally, the close relation between the multifaceted chiral properties of the Fe langasites and their multiferroic potential is expected to motivate further studies.

We would like to acknowledge A. Hadj-Azzem and J. Debray for their help in the preparation of the samples and P. Bordet, S. Grenier, and O. Cépas for fruitful discussions. This work was financially supported by Grant No. ANR-13BS04-0013. 
[1] Y. Tokura, S. Seki, and N. Nagaosa, Rep. Prog. Phys. 77, 076501 (2014).

[2] S. Dong, J.-M. Liu, S.-W. Cheong, and Z. Ren, Adv. Phys. 64 519 (2015).

[3] D. I. Khomskii, arXiv:1510.05174.

[4] E. Bousquet and A. Cano, J. Phys.: Condens. Matter 28, 123001 (2016).

[5] B. V. Mill', E. L. Belokoneva, and T. Fukuda, Russian Journal of Inorganic Chemistry 43, 1168 (1998).

[6] K. Marty, V. Simonet, E. Ressouche, R. Ballou, P. Lejay, and P. Bordet, Phys. Rev. Lett. 101, 247201 (2008).

[7] V. Simonet, M. Loire, and R. Ballou, Eur. Phys. J. Special Topics 213, 5 (2012).

[8] V. Yu Ivanov, A. A. Mukhin, A. S. Prokhorov, and B. V. Mill', Solid State Phenomena 152, 299 (2009).

[9] K. Marty, P. Bordet, V. Simonet, M. Loire, R. Ballou, C. Darie, J. Kljun, P. Bonville, O. Isnard, P. Lejay et al., Phys. Rev. B 81, 054416 (2010).

[10] L. Chaix, S. de Brion, F. Lévy-Bertrand, V. Simonet, R. Ballou, B. Canals, P. Lejay, J. B. Brubach, G. Creff, F. Willaert et al., Phys. Rev. Lett. 110, 157208 (2013).

[11] H. D. Zhou, L. L. Lumata, P. L. Kuhns, A. P. Reyes, E. S. Choi, N. S. Dalal, J. Lu, Y. J. Jo, L. Balicas, J. S. Brooks et al., Chem. Mater. 21, 156 (2009).

[12] N. Lee, Y. J. Choi, and S.-W. Cheong, Appl. Phys. Lett. 104, 072904 (2014).

[13] I. A. Sergienko and E. Dagotto, Phys. Rev. B 73, 094434 (2006).

[14] H. Katsura, N. Nagaosa, and A. V. Balatsky, Phys. Rev. Lett. 95, 057205 (2005).

[15] M. Mostovoy, Phys. Rev. Lett. 96, 067601 (2006).

[16] J. S. White, C. Niedermayer, G. Gasparovic, C. Broholm, J. M. S. Park, A. Y. Shapiro, L. A. Demianets, and M. Kenzelmann, Phys. Rev. B 88, 060409 (2013).

[17] K. Cao, R. D. Johnson, F. Giustino, P. G. Radaelli, G.-C. Guo, and L. He, Phys. Rev. B 90, 024402 (2014).
[18] T.-h. Arima, J. Phys. Soc. Jpn. 76, 073702 (2007).

[19] M. Kenzelmann, G. Lawes, A. B. Harris, G. Gasparovic, C. Broholm, A. P. Ramirez, G. A. Jorge, M. Jaime, S. Park, Q. Huang et al., Phys. Rev. Lett. 98, 267205 (2007).

[20] L. N. Bulaevskii, C. D. Batista, M. V. Mostovoy, and D. I. Khomskii, Phys. Rev. B 78, 024402 (2008).

[21] D. I. Khomskii, J. Phys.: Condens. Matter 22, 164209 (2010).

[22] M. Loire, V. Simonet, S. Petit, K. Marty, P. Bordet, P. Lejay, J. Ollivier, M. Enderle, P. Steffens, E. Ressouche et al., Phys. Rev. Lett. 106, 207201 (2011).

[23] A. Zorko, M. Pregelj, A. Potocnik, J. van Tol, A. Ozarowski, V. Simonet, P. Lejay, S. Petit, and R. Ballou, Phys. Rev. Lett. 107, 257203 (2011).

[24] J. Jensen, Phys. Rev. B 84, 104405 (2011).

[25] See Supplemental Material at http://link.aps.org/supplemental/ 10.1103/PhysRevB.93.214419 for more information about magnetic structure factor, chirality, neutron polarimetry, electron spin resonance, and calculations.

[26] C. Stock, L. C. Chapon, A. Schneidewind, Y. Su, P. G. Radaelli, D. F. McMorrow, A. Bombardi, N. Lee, and S.-W. Cheong, Phys. Rev. B 83, 104426 (2011).

[27] I. S. Lyubutin, P. G. Naumov, B. V. Mill', K. V. Frolov, and E. I. Demikhov, Phys. Rev. B 84, 214425 (2011).

[28] B. Coqblin, The Electronic Structure of Rare Earth Metals and Alloys (Academic, New York, 1978).

[29] J. Jensen and A. R. Mackintosh, Rare Earth Magnetism (Clarendon, Oxford, 1991)

[30] V. Scagnoli, S. W. Huang, M. Garganourakis, R. A. de Souza, U. Staub, V. Simonet, P. Lejay, and R. Ballou, Phys. Rev. B 88, 104417 (2013).

[31] C. Toulouse, M. Cazayous, S. de Brion, F. Levy-Bertrand, H. Barkaoui, P. Lejay, L. Chaix, M. B. Lepetit, J. B. Brubach, and P. Roy, Phys. Rev. B 92, 104302 (2015).

[32] For unbalanced ferroelectric domain populations.

[33] C. Lee, E. Kan, H. Xiang, and M.-H. Whangbo, Chem. Mater 22, 5290 (2010). 